\title{
Semantic Interoperability in Heterogeneous IoT Infrastructure for Healthcare
}

\author{
Sohail Jabbar, ${ }^{1}$ Farhan Ullah, ${ }^{2}$ Shehzad Khalid, ${ }^{3}$ Murad Khan, ${ }^{1}$ and Kijun Han ${ }^{1}$ \\ ${ }^{1}$ School of Computer Science and Engineering, Kyungpook National University, Daegu, Republic of Korea \\ ${ }^{2}$ Department of Computer Science, COMSATS Institute of Information Technology, Sahiwal, Pakistan \\ ${ }^{3}$ Department of Computer Engineering, Bahria University, Islamabad, Pakistan \\ Correspondence should be addressed to Kijun Han; kjhan@knu.ac.kr
}

Received 21 December 2016; Accepted 6 February 2017; Published 5 March 2017

Academic Editor: Yoshikazu Miyanaga

Copyright @ 2017 Sohail Jabbar et al. This is an open access article distributed under the Creative Commons Attribution License, which permits unrestricted use, distribution, and reproduction in any medium, provided the original work is properly cited.

Interoperability remains a significant burden to the developers of Internet of Things' Systems. This is due to the fact that the IoT devices are highly heterogeneous in terms of underlying communication protocols, data formats, and technologies. Secondly due to lack of worldwide acceptable standards, interoperability tools remain limited. In this paper, we proposed an IoT based Semantic Interoperability Model (IoT-SIM) to provide Semantic Interoperability among heterogeneous IoT devices in healthcare domain. Physicians communicate their patients with heterogeneous IoT devices to monitor their current health status. Information between physician and patient is semantically annotated and communicated in a meaningful way. A lightweight model for semantic annotation of data using heterogeneous devices in IoT is proposed to provide annotations for data. Resource Description Framework (RDF) is a semantic web framework that is used to relate things using triples to make it semantically meaningful. RDF annotated patients' data has made it semantically interoperable. SPARQL query is used to extract records from RDF graph. For simulation of system, we used Tableau, Gruff-6.2.0, and Mysql tools.

\section{Introduction}

Internet of things (IoT) is a combination of spatially distributed smart objects which have sensing capabilities and embedded identification through RFID technology. Specifically, the integration of sensors, RFID tags, and communicating technologies forms the underpinning of IoT. It addresses the traceability, visibility, and controllability of smart objects. It is a future vision through which digital and physical objects can be interlinked and intercommunicated to provide some domain specific services. IoT transforms real world objects into smart objects which can sense the environmental physical quantity and communicate it accordingly. RFID technology is now widely used in tracking of people, objects, and animals. Electronic Product Codes are encoded in RFID tags which can be used to track smart objects in IoT $[1,2]$. For storage of incoming data from these smart devices, Cloud technology is one of the suitable options. There is a long list of its applications like environmental monitoring [3], healthcare service [4], inventory and production management [5], food supply chain [6], transportation and logistics [7], smart cities [8], smart homes [9], data collection through aerial vehicles [10], firefighting system [11], social networks [12], and VANETs [13] just to name a few.

In spite of its wide spread emergence, IoT is still in its infant stage and has big room for research in variety of issues like standards, scalability, heterogeneity of different devices, common service description language, domain specific service discovery, integration with existing IT systems, and so forth. In IoT, one vital issue is interoperability among smart objects that is the ability to interconnect and communicate different vendors' systems to form a cost effective and easy to implement network. To promote enterprise interoperability, C4ISR architecture working group (AWG) developed the LISI (Levels of Information Systems Interoperability) approach in 1997 [14]. The purpose of LISI model is to provide maturity model to US Department of Defense (DoD) and to specify interoperability needs and selecting pragmatic solutions for enterprise interoperability [15]. IoT industry is working to migrate the interoperability standards to existing 
IP and Ethernet standards to work with existing infrastructure [16]. According to Cisco analysis, there will be 50 billion connected devices [17]. The IoT Forums from worldwide are working to develop a common model that can ensure interoperability among smart objects. Open Internet Consortium (OIC) is currently focusing on the IoT interoperability to define specifications, integration of billions of smart objects, and scalability issues (http://blogs.cisco.com/digital/iotmeets-standards-driving-interoperability-and-adoption). Low cost interoperability among smart objects is an important factor for smart cities. Smart mesh backbone gateways (WRT54GL by Linksys (http://www.linksys.com/us/supportproduct?pid $=01 \mathrm{t} 80000003 \mathrm{KOkNAAW})$ and net5501 by Soekris Engineering Inc. (http://soekris.com/products/eol-products/net5501.html)) are developed for smart cities which provide low cost interoperability. The Grid-Wise Architecture Council (GWAC) mission is to enable interoperability among the objects that interact with the electric power system. The GWAC introduced a context setting framework which identifies interoperability issues [1]. The solution of interoperability will help the customers to continue working in mixed environments in nearer future. It will solve the complexity of an organization's infrastructure, reduce the cost of buildings, and help in supporting heterogeneous infrastructure. Energy aware algorithms will be required to ask from users for routing their packets on wireless or IEEE 802.11 mesh network. Thus, it will save the energy of smart objects [18]. Users with multiple vendors' technologies from worldwide bring scalability in IoT. It will increase the workflow efficiency in any environment. It will connect objects from anywhere to anywhere on different technologies.

A productive effort in developing widely accepted standards can smoothly lead to solving the interoperability issue. The development of standards is for ease of plug and play device interaction, reconfigurability, and information exchange. All smart objects from multiple vendors should operate on common standards. If we can integrate IoT with existing IT infrastructure then it will solve many problems like protocol, packet size, encryption/decryption algorithms, and so forth. Operating smart objects to existing Internet Protocol version 6 (IPv6) structure may be costly because it is difficult to embed RJ45/WLAN port in every smart object but IoT industry is currently working to migrate the IoT standards to existing IPv6 and Ethernet standards to ensure the interoperability with legacy protocols. IP for Smart Objects (IPSO) (http://www.ipso-alliance.org) promotes IPv6 as a unique identification for discovering, accessing, and communicating smart objects [15]. Secondly in case of device roaming, a specific device identity is required and not IP address. Therefore every device must need a unique identification worldwide.

Collaboration among different vendors can also be a workable effort towards solving interoperability issues. Vendors should collaborate on these issues and develop a transparent method for interoperability that can be implemented in every domain. Collaboration is needed between enterprise modeling communities in developing business oriented architecture and software engineering companies working on IT oriented solutions [15]. Interoperability methods should not be domain specific. Interoperability can be solved if communicating smart objects are semantically interoperable [19]. Semantics brings what data is transferring. It gives the details about structure of transferred data. IoT device's semantics details, interpretation, and exchange of information must be developed in order to remove semantics conflicts in interoperability. The meaning responding from a smart object is fully interpretable as originally means by the smart object $[2,20]$. Strong infrastructure is needed for interoperability to fit any device from multiple vendors. Consumer should feel free to use any device from any vendor and vendor should take care of consumer trust. Interoperability has a major issue of global heterogeneity because IoT devices are highly heterogeneous. A huge number of IoT devices from unknown vendors have different configuration and installation methods. Devices from multiple vendors have different methods for semantics and syntactic interoperability methods, so there will be semantic and syntactic conflicts. It will be difficult to add a new device in IoT network without resolving semantic ambiguity. In healthcare domain it can provide high quality care to patients when every device works in "interoperable environment." In healthcare industry it will automate the hospitals that would monitor the patients by physicians remotely. Physicians could get information about patients quickly and advise them for treatment. It will provide fast access to patients' records from different IT storage systems located anywhere.

In this research work, Resource Description Framework is used to provide Semantic Interoperability among physicians and patients using heterogeneous IoT devices. We have analyzed dataset on proposed IoT based Semantic Interoperability Model to provide interoperability in IoT devices. Patients' dataset semantically annotated to convert it to RDF and SPARQL query is used to extract data of any patient at any time. In proposed model medicines' data of pharma companies stored on Cloud and Physicians can access information due to semantics interoperability. Collected data is transformed into linked data (RDF triples) and SPARQL queries are applied to extract patient related semantic information from that linked data. A similar work is done by Malik et al. in [21]. A lightweight model for semantic annotation of data using heterogeneous devices in IoT has been proposed to make IoT devices interoperable. Communicating data between physician and patient is semantically annotated which solve the issue of interoperability among IoT devices.

This paper is further divided into 5 sections: Literature Review, Proposed Scheme: IoT Based Semantic Interoperability Model (IoT-SIM), Semantic Annotations of Data Using Heterogeneous IoT Devices, Experiments and Results, and Conclusion and Future Work.

\section{Literature Review}

IoT devices from different vendors can be integrated in healthcare environment to assist Physicians and patients remotely. Physicians can monitor their patients anytime and anywhere and can update prescription when needed. In [22], the authors used Intelligent Personal Assistant (IPA) 
as software agent in IoT device for physician to give real time information about the monitored patients. The AMBRO mobile gateway collects information from different IPA devices and then take action(s). It enabled interoperability among different IPA devices. It uses only specific operating system and does not integrate with any other operating system such as $\mathrm{iOS}$ or windows phone. In order to communicate large number of smart devices with heterogeneous capabilities, semantic web technologies are promising tools for this purpose to share data and exchange their services efficiently. In [23], the authors proposed a semantic model for description of smart objects using ontologies and description logics to enable Semantic Interoperability. This semantic model still needs further empirical evaluation to enhance the service classifications for Semantic Interoperability among smart objects. To digitize agriculture domain, there is a need to collect information about crop growth monitoring and irrigation decisions support through smart objects.

Jayaraman et al. [24] described an OpenIoT platform used for digital agriculture use case (Phenonet). To enable Semantic Interoperability, OpenIoT platform used ontologies to represent Phenonet domain concepts to collect smart collection of information, annotation, and validation processes. A scalable and intelligent IoT architecture is required for future era to enable discovery of physical sensors and their interoperation semantically and syntactically. A semantic web enabled architecture is proposed by Desai et al. [25] to provide interoperability among smart things. The Semantic Gateway as Service (SGS) integrated semantic web technologies to enable communication between protocols such as XMPP, CoAP, and MQTT. The ontologies are used for semantic reasoning to provide Semantic Interoperability among communicating messages. Although there are different approaches developed for semantic interoperability for IoT devices, there is still lack of formal methodologies for interoperability in technology and standard format of data.

Gyrard and Serrano [26] proposed SEG 3.0 methodology to unify, federate, and provide Semantic Interoperability in IoT domain. The SEG 3.0 emerged from ontology engineering and its main benefit to integrate heterogeneous data collected from different smart things. The authors applied SEG 3.0 methodology on three different use cases, in M3 framework to support developers to design semantic-based IoT applications, the VITAL EU project for smart cities, and the FIESTA-IoT EU project for Semantic Interoperability. For satisfying level of performance among low-power heterogeneous networks, there is a need of interoperability protocols and standards. To bridge this gap, the authors in [27] described features of different protocol stacks such as Bluetooth, Bluetooth Low Energy, IEEE 802.15.4, ZigBee, 6LoWPAN, and IEEE 802.15.6. They proposed the designing of generic protocol stack that communicates with multiple radios and with different protocols simultaneously, regardless of IP-based or non IP-based networks. Mingozzi et al. [28] proposed a novel uniform platform designed to include context-awareness functionalities and showed how such functions can be exploited to automate search and selection of things through natural language. It is shown that context can be used on things services to extract knowledge through semantic reasoning in smart homes. Today's smart phones have vast connectivity and sensing capabilities for serving body area networks and physiologic sensors. To enable interoperability between Machine-to-Machine (M2M) and IoT, common standards are the driving needs. In this context, a design of ETSI M2M gateway integrated with libraries is proposed by Pereira et al. [29]. This is to ease the deployment of IoT applications with reduced development costs. Its performance is shown by measuring the smartphone's CPU and memory usage and battery life. In [30], authors proposed an architecture for data processing in IoT Cloud environment that supports semantics interoperability. Google Cloud and Microsoft Azure were used as multi-Cloud environment with OpenIoT architecture. Another IoT concept for digitizing the factory is proposed in [31]. Data communicates from heterogeneous IT environment to ensure data consistency. Author suggestion is the integration of IoT and PLM platforms using semantic web technologies and Open Services for Lifecycle Collaboration (OSLC) standard on tool interoperability.

\section{Proposed Scheme: IoT Based Semantic Interoperability Model}

In our proposed model we focused on tracking and monitoring of human diseases with respect to prescribed medicine in healthcare domain. User Interface (UI), Semantic Interoperability (SI), and Cloud Services (CS) are three major constituents of IoT-SIM. In User Interface, doctor and patient interact with each other with the help of IoT devices. Doctor can monitor and prescribe patients remotely anywhere, anytime, and without any constraint of specific vendors' device. Semantic Interoperability directly interacts with User Interface section. Interoperability among IoT devices from different vendors is a big issue. Semantic Interoperability is the exchange of information with meaningful and understandable meanings. It includes semantics in data by adding self-described information packages. Semantic Interoperability is used to ensure that IoT devices from different vendors are interoperable. IoT devices take data from UI and then add semantic annotations from semantic section to make it meaningful with shared vocabulary. Data analytics is a data science technique which draws meaningful conclusions from raw data. It brings cost reduction and better and fast decision making. Data analytics technique is applied on collected data from IoT devices. Then, it underwent semantic annotations to make it more meaningful and cost effective. The proposed architectural model for semantic interoperability in IoT is shown in Figure 1.

\subsection{Semantic Annotations of Data Using Heterogeneous IoT} Devices. IoT devices are communicating through sensors. Every device has sensor network API which is used to filter data according to the domain. Then this filtered data is sent to sensors' web service through which sensors are communicating with the whole world. To provide web services using heterogenous IoT devices Sensor Web Enablement framework has been used. SWE used to discover, access, and use IoT devices. These keywords are tokens which represent healthcare data related to human diseases. Then dataset is 


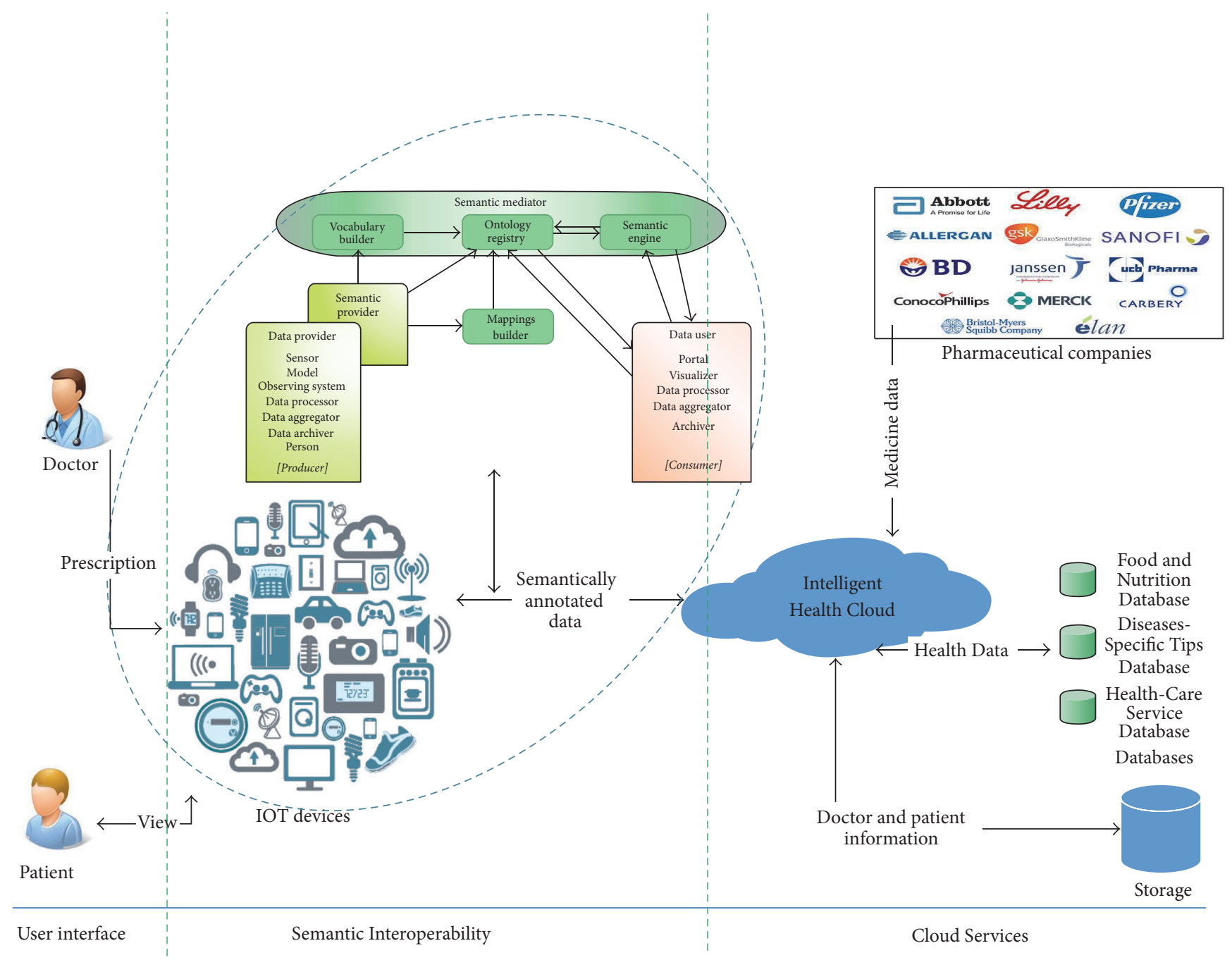

FIgURE 1: Architectural model for Semantic Interoperability in IoT.

moved to sematic interoperability section where every token is categorized in specific domain of hum diseases. Every token with its description is sent to diseases classification section. In this section human disease is classified to show which patient has which type of disease. A lightweight model for semantic annotation of data using heterogeneous devices in IoT that is depicted in Figure 2 represents this operational flow from data collection to classification of patients according to their diseases.

On identification of disease, system can automatically suggest medicine for it. If prescribed medicine from doctor to patient is matched with identified disease then it is correct medicine otherwise doctor prescribed the wrong medicine. Storage attached with Intelligent Health Cloud stores information about correct or wrong medicine with doctors' and patient's identification. Classified diseases in different categories according to healthcare domain are sent to Tagging section where diseases are semantically annotated automatically or manually through Resource Description Framework. RDF is a standard metadata model that uses
URIs (Uniform Resource Identifier) to relate things semantically. The same is also shown in Figure 2.

\section{Experiments and Results}

We have analyzed our experiments on Heart Disease Dataset (Heart Disease Dataset from Center for Machine Learning and Intelligent Systems: https://archive.ics.uci.edu/ml/ datasets/Heart+Disease). This dataset is collected from University Hospital, Zurich, Switzerland (switzerland.data). The principal investigator responsible for the data collection is VA Medical Center, Long Beach and Cleveland Clinic Foundation, Robert Detrano, M.D., Ph.D. The dataset contains 123 patients with different types of heart diseases. Dataset is plotted graphically with detailed information as shown in Figure 3. This graph shows that dataset consists of patient Id, age, sex, and disease type. For sex attribute, 1 denotes male and 0 denotes female while for all other attributes, 1 denotes yes and 0 denotes not. There are 14 different attributes used for a patient in a database. Dig attribute describes Digitalis used during exercise and its value is 1 or 0 . 


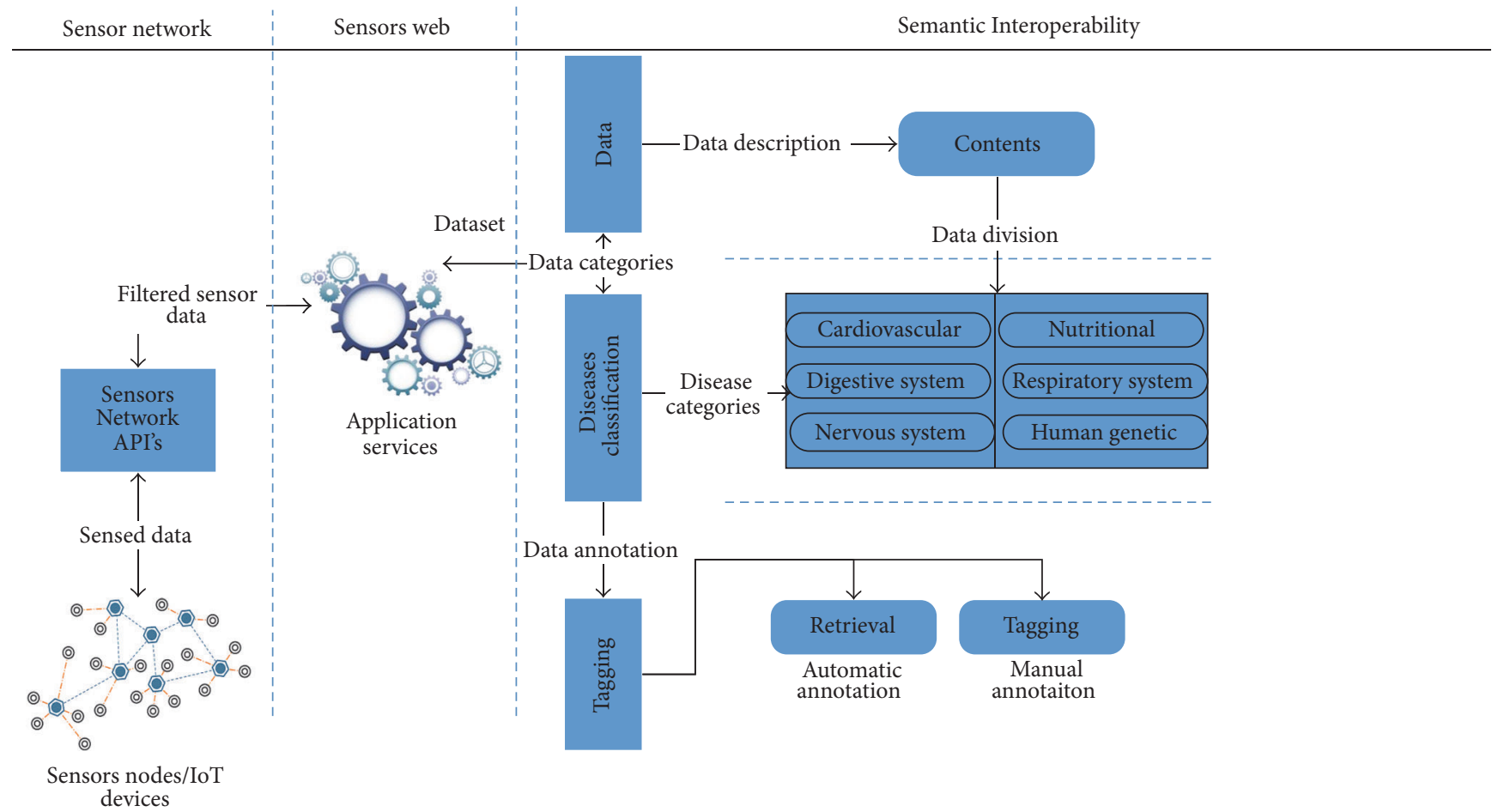

FIGURE 2: Lightweight Model for Semantic annotation of data using heterogeneous devices in IoT.

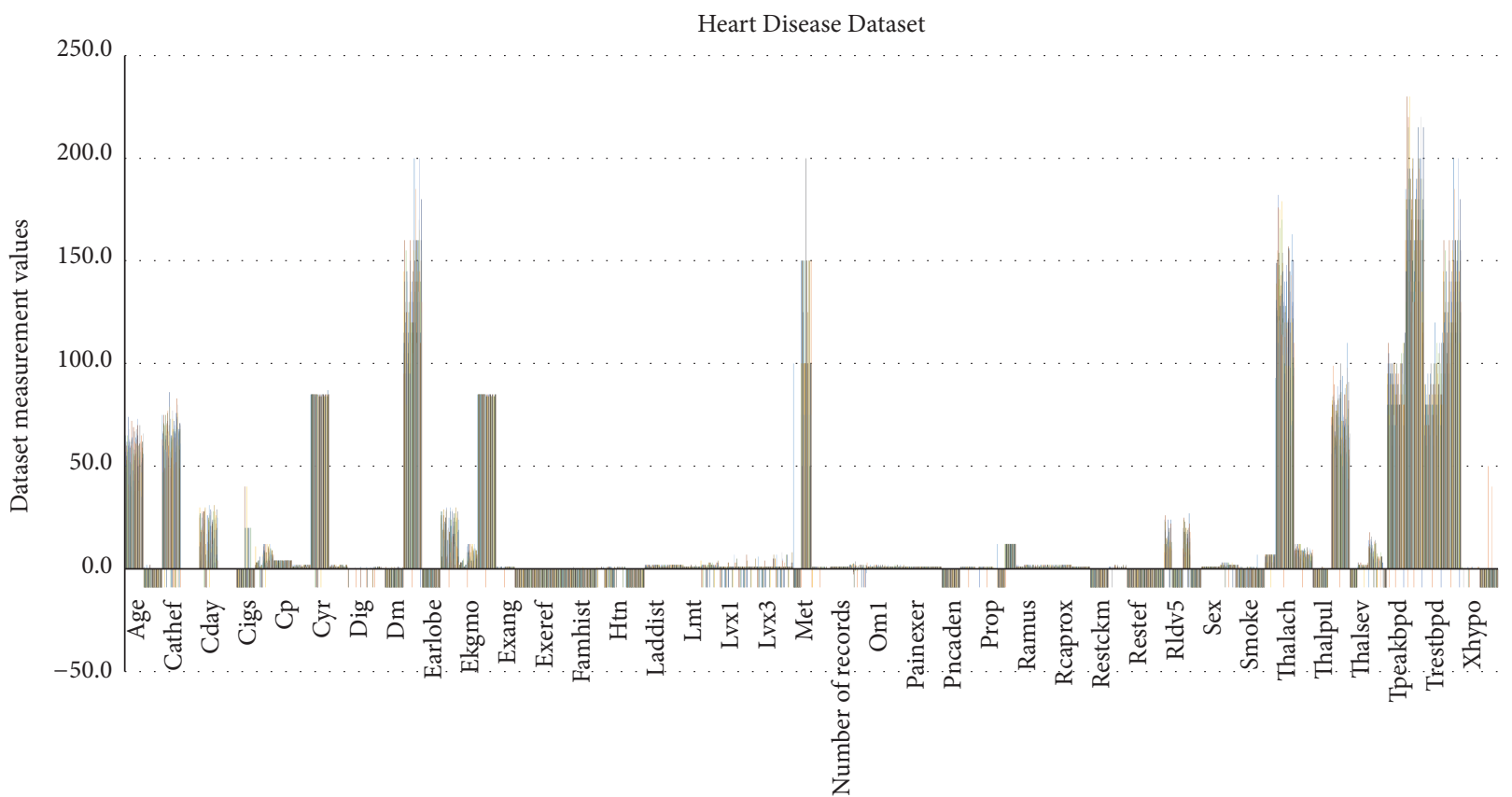

Patient attributes used for data collection from IoT or non-IoT devices

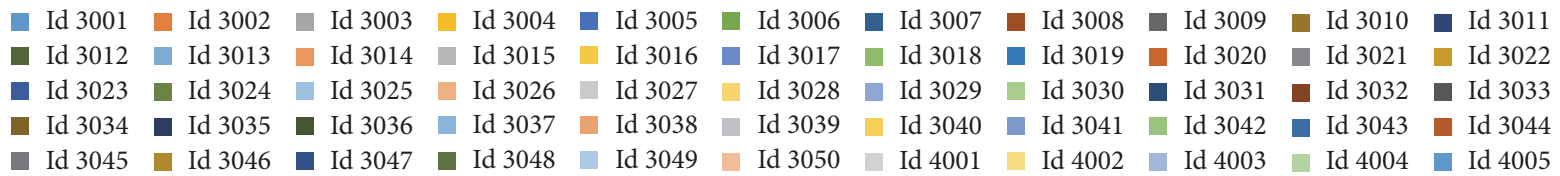

FIGURE 3: Patient Id-Wise dataset graph representation. 


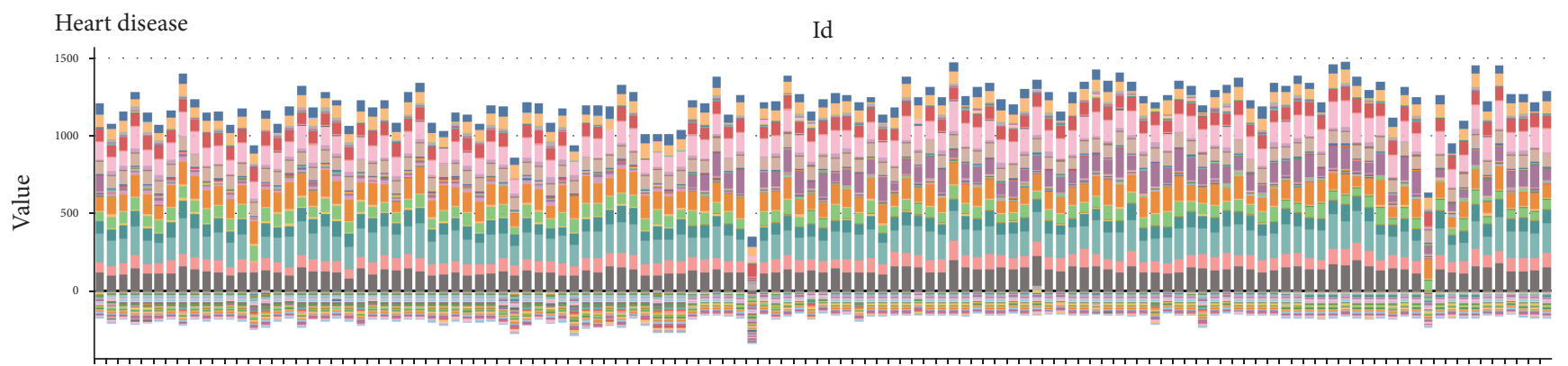

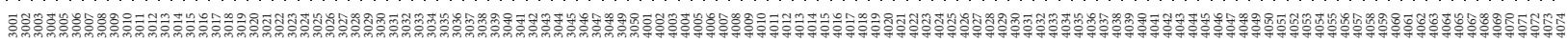

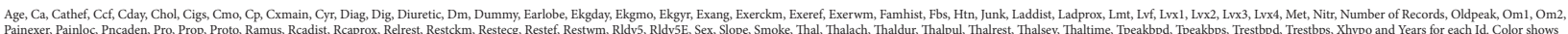

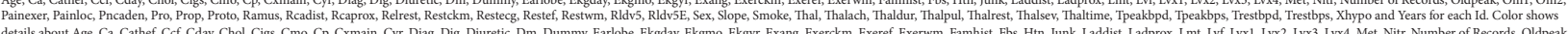

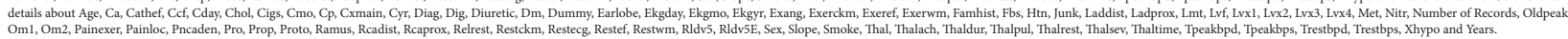

\begin{tabular}{|c|c|}
\hline Age & Exerckm \\
\hline$\| \mathrm{Ca}$ & Exeref \\
\hline II Cathef & Exerwm \\
\hline || Ccf & Famhist \\
\hline Cday & $\mathrm{Fbs}$ \\
\hline I Chol & Htn \\
\hline IIgs & I Junk \\
\hline $\mathrm{Cmo}$ & Laddist \\
\hline Cp & Ladprox \\
\hline Cxmain & Lmt \\
\hline Cyr & Lvf \\
\hline
\end{tabular}

1 Painexer
- Painloc
1 Prcaden
- Pro
- Proto
1 Ramus
- Rcadist
- Rcaprox
- Relrest
- Restckm

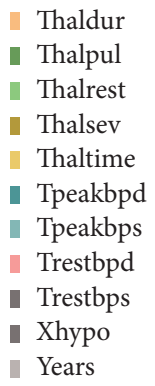

Diag

I Dig

Ivx1

|1 Diuretic Ivx3

I Dm Lvx4

॥ Dummy Met

- Earlobe

- Ekgday

- Ekgmo

II Ekgyr

- Exang

Nitr

I Number of Records

- Oldpeak

I Om1

| Om2

I Restecg

1. Restef

1. Restwm

- Rldv5

Rldv5E

I Sex

- Slope

I Smoke

Thal

- Thalach

Figure 4: Accumulated heart diagnostic measurements.

All attributes are plotted horizontally and its corresponding weighted value is plotted vertically. Mostly patients are 60 to 65 years of age as shown in age attribute. In graph Tpeakbps has highest value meaning that almost all heart patients have blood pressure disease as well. Some attributes are negative in the graph. It intuits that these attributes are not used during experiment as shown in Figure 3. We used Tableau tool (http://www.tableau.com/products/desktop) to plot patients' accumulated graph against their diseases. The patients identified by their Ids are plotted at $x$-axis and accumulated values of their diseases are plotted at $x$-axis. Patients' attributes are used for data collected from IoT or non-IoT devices as shown in Figure 3.

Accumulated heart diagnostic measurements of dataset are plotted as shown in Figure 4. Figure 4 is the complete reflection of dataset using accumulated measurements of attributes in dataset which showed that the collected dataset is fully correct and validate. There are different colors used to identify diseases. Many attributes are negative of $4006 \mathrm{Id}$ patient which shows that mostly diseases are not analyzed during experiment of this patient. Tpeakbps value shows in a graph almost all patients' diagnosis blood pressure disease as well. Some patients like 3014, 4006, and 4063 have Tpeakbps value in negative shows that blood pressure is not analyzed during experiment. Patients with 4023 and 4056 have diagnosis of many diseases and have almost the same number of diseases. Cigs defines cigarettes per day and its value is negative means that it is not considered during experiment. The names and social security numbers of the patients were recently removed from the database, replaced with dummy values. In this paper we have simulated our proposed IoT-SIM to provide Semantic Interoperability among heterogeneous IoT devices through RDF to make it readable by machine and human. In [32], RDF is graphical database which assign Universal Resource Identifier (URI) for identification. Further author used ontologies to identify the same entity with different meanings. We annotated semantically all the data to make triples which shows relationship among every instance. Every instance in triples is identified by some URI and every instance relates with other instances using some semantic relation.

In Figure 5 the RDF graph is simulated from patients' database in Gruff-6.2.0 tool (http://franz.com/agraph/gruff/ release-history.lhtml). Every attribute has specific color for its identification. Info is a table which relates with every instance semantically and all Info nodes in RDF graph relate to only one Info table. All fields are semantically annotated using $\mathrm{RDF}$ triples, which shows the relation of every instance with other related instances. Patients' records against diagnostics diseases are semantically readable by machine to ensure interoperability among heterogeneous IoT devices. In [33], SPARQL, a structural query language proposed by W3C, is designed for querying RDF data. Since SPARQL queries can be represented as query graphs, a SPARQL query can be answered by performing the graph pattern matching over RDF graphs. Figure 5's different visible chunks are shown in Figure 6 which shows single node RDF graph interaction with other node. A single node interaction with different attributes in dataset using RDF graph is shown in Figure 6. All detailed information of all patients is semantically annotated using RDF graph to provide matching of information semantically ignoring the vendors' hardware details. Further to validated 


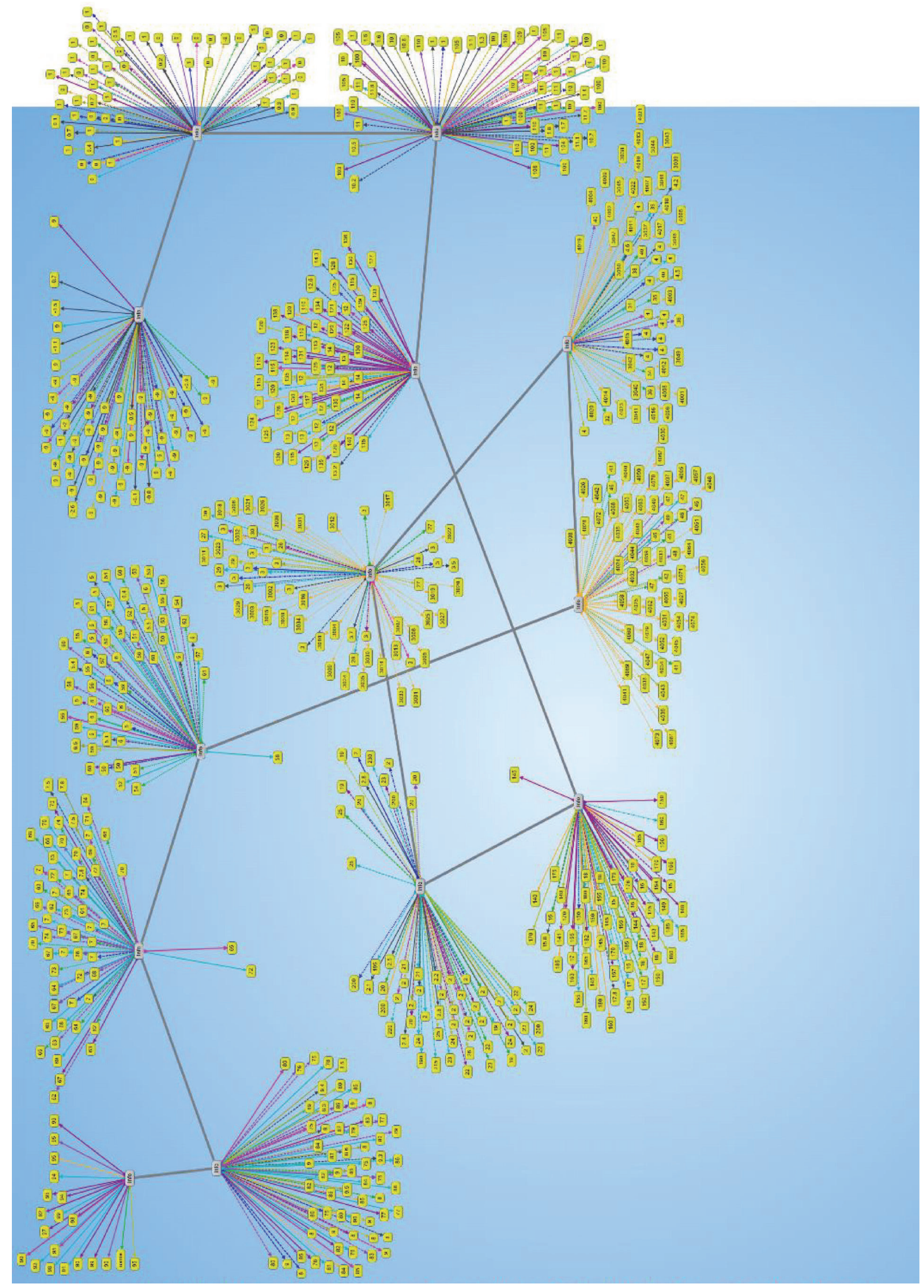

FIGURE 5: RDF graph for Semantic Interoperability in IoT. 


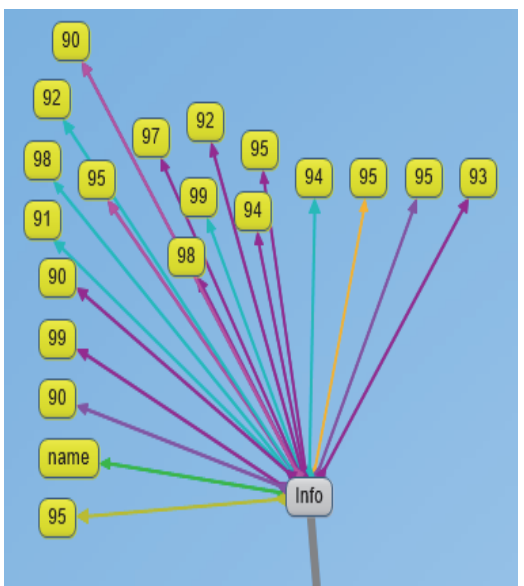

(a)

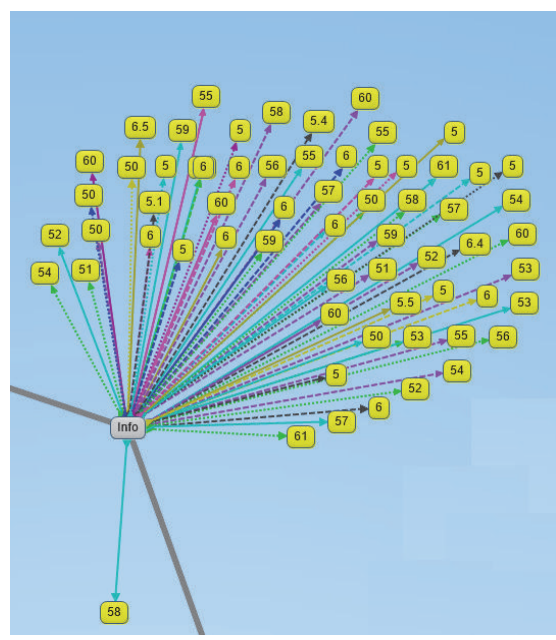

(d)

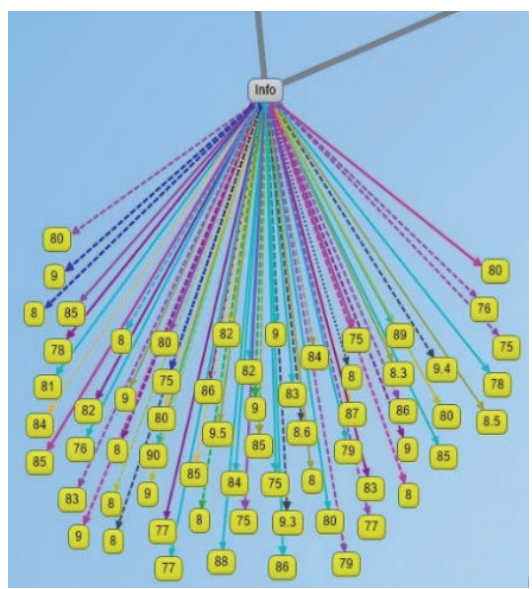

(b)

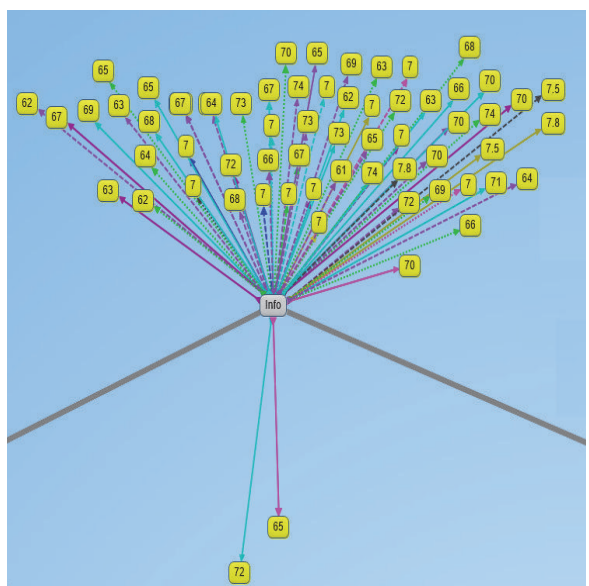

(c)

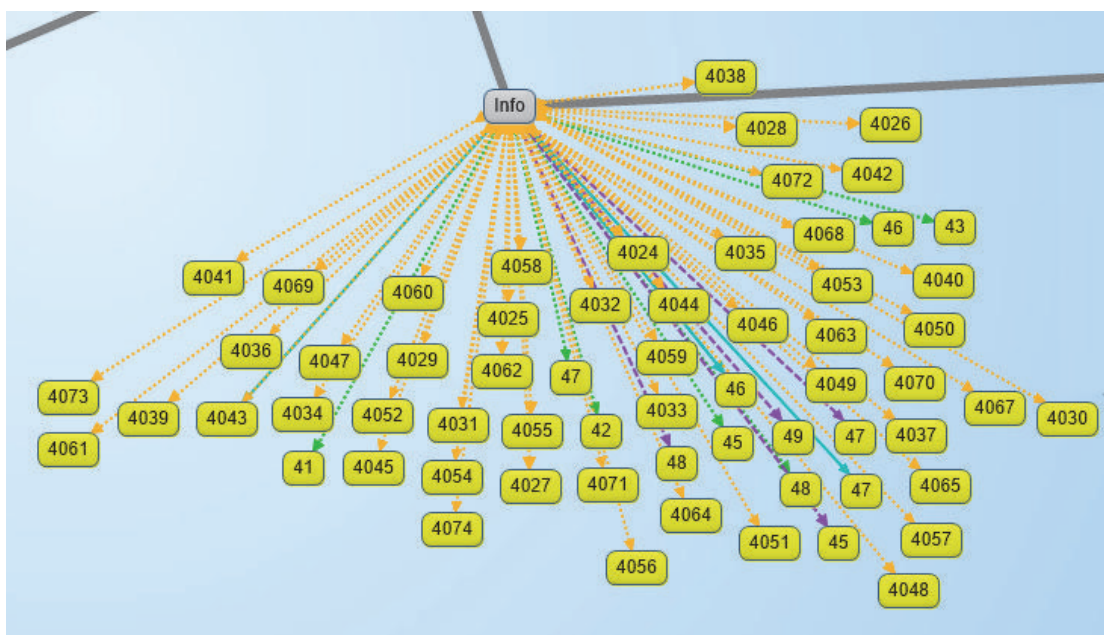

(e)

\begin{tabular}{|c|c|c|c|c|}
\hline Age & Ekgday & Lvx2 & Rcadist & Thalpul \\
\hline (2) & Ekgmo & Lvx3 & Rcaprox & 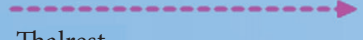 \\
\hline Cathef & Ekgyr & Lvx4 & $\cdots$ & mairest \\
\hline Cef & 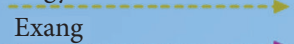 & Met & Kentest & Thalsev \\
\hline Cday & Exerckm & Name & Restckm & Thaltime \\
\hline Chol & Exeref & Nitr & Restecg & Tpeakbpd \\
\hline Cigs & Exerwm & Num & Restef & Tpeakbps \\
\hline $\mathrm{Cmo}$ & Famhist & Oldpeak & Restwm & \\
\hline $\mathrm{Cp}$ & Fbs & $\mathrm{Om} 1$ & Rldv5 & 110 cosu \\
\hline Cxmain & Htn & $\mathrm{Om} 2$ & Rldv5e & Trestbps \\
\hline Cyr & Id & Painexer & & Xhypo \\
\hline Diag & Junk & Painloc & Slope & Years \\
\hline Dig & Laddist & Pncaden & $\rightarrow$ & \\
\hline $\mathrm{Dm}$ & $\mathrm{Lmt}$ & Prop & Thal & Literal \\
\hline Dummy & Lvf & Proto & Thalach & \\
\hline Earlobe & (..................... & Ramus & Thaldur & noty \\
\hline
\end{tabular}

(f)

FIGURE 6: RDF graph chunks for Semantic Interoperability in IoT. 
our experiment, we extracted records of different patients from RDF graph using SPARQL query in a text format.

The SPARQL query has been used to extract all patients records from $\mathrm{RDF}$ graph as $\mathrm{RDF}$ is semantically machine readable. We can also extract information of any patient using SPARQL query.

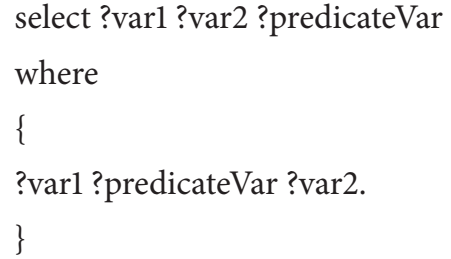

The visible chunks of simulated graph in Figure 5 are shown in Figure 6.

The attributes/legends of patients' database in RDF graph are shown in Figure 6(f). Every attribute has different color to identify in RDF graph. For example, age attribute/legend has light green color which shows every patient's age in RDF graph. These legends describe attributes in dataset which relates with patients' nodes in terms of semantically annotating.

\section{Conclusion and Future Work}

In this paper, we have proposed an IoT-SIM model for Semantic Interoperability among heterogeneous IoT devices in healthcare domain. The main goal of this model is to provide interoperability among heterogenous IoT devices by using semantically annotated. Physicians monitor their patients remotely anywhere, anytime, and without any constraint of specific vendors' device. RDF is used to present patients' raw data into useful information. Physicians prescribed patients against the diagnosis diseases using IoT device; then this information is semantically annotated using RDF. Further lightweight model for semantic annotation of data using heterogeneous devices in IoT has been proposed which is describing the communication among heterogenous IoT devices. To provide web services, sensors' devices communicated using SWE framework with other devices. To solve the issue of interoperability among heterogeneous IoT devices, collected dataset has been mapped to RDF graph database. The communicated data is being analyzed and annotated in terms of semantics. Annotated information is then sent to Intelligent Health Cloud where prescribed medicines match from pharmaceutical companies, then prescribed information with medicine details is sent to patient IoT. RDF graph represents patients' diseases database in triples which is semantically readable using SPARQL query. Physicians can query from his IoT device any time about the current situation of the patient from database remotely. End users do not care for time, distance, and hardware.

Our proposed model can be enhanced in future to provide syntactic interoperability among heterogeneous IoT devices. Syntactic interoperability provides the syntactic structure of the exchanged information. Next security is a hot issue in interoperability among heterogeneous IoT devices. It must be critical aspect for the solution of interoperability.
The syntactic interoperability and security issues will be investigated in the future of this work.

\section{Competing Interests}

The authors declare that they have no competing interests.

\section{Acknowledgments}

This research was supported by Basic Science Research Program through the National Research Foundation of Korea (NRF) funded by the Ministry of Education (2016R1D1A1B03933566). This study was supported by the BK21 Plus project (SW Human Resource Development Program for Supporting Smart Life) funded by the Ministry of Education, School of Computer Science and Engineering, Kyungpook National University, Korea (21A20131600005).

\section{References}

[1] S. L. Hamilton, E. W. Gunther, R. V. Drummond, and S. E. Widergren, "Interoperability-a key element for the grid and der of the future," in Proceedings of the IEEE Power Engineering Society Transmission and Distribution Conference, pp. 927-931, Dallas, Tex, USA, May 2006.

[2] G. Xiao, J. Guo, L. D. Xu, and Z. Gong, "User interoperability with heterogeneous IoT devices through transformation," IEEE Transactions on Industrial Informatics, vol. 10, no. 2, pp. 14861496, 2014.

[3] D. Pavithra and R. Balakrishnan, "IoT based monitoring and control system for home automation," in Proceedings of the Global Conference on Communication Technologies (GCCT '15), pp. 169-173, IEEE, Kanyakumari, India, April 2015.

[4] B. R. Nugroho, "The architecture of an IoT-based healthcare monitoring system using smart e-health gateways in home/ hospital domain," Buletin Inovasi ICT \& Ilmu Komputer, vol. 2, no. 1, 2015.

[5] A. Agra, M. Christiansen, K. S. Ivarsøy, I. E. Solhaug, and A. Tomasgard, "Combined ship routing and inventory management in the salmon farming industry," Annals of Operations Research, pp. 1-25, 2016.

[6] X. Zhao, H. Fan, H. Zhu, Z. Fu, and H. Fu, "The design of the internet of things solution for food supply chain," in Proceedings of the International Conference on Education, Management, Information and Medicine, Shenyang, China, April 2015.

[7] S. Jabbar, M. Khan, B. Nathali Silva, and K. Han, "A REST-based industrial web of things' framework for smart warehousing," The Journal of Supercomputing, 2016.

[8] P. Misra, V. Rajaraman, K. Dhotrad, J. Warrior, and Y. Simmhan, "An interoperable realization of smart cities with plug and play based device management," 2015, https://arxiv.org/abs/1503 .00923 .

[9] M. Khan, S. Din, S. Jabbar, M. Gohar, H. Ghayvat, and S. C. Mukhopadhyay, "Context-aware low power intelligent SmartHome based on the Internet of things," Computers \& Electrical Engineering, vol. 52, pp. 208-222, 2016.

[10] O. Aldabbas, A. Abuarqoub, M. Hammoudeh, U. Raza, and A. Bounceur, "Unmanned ground vehicle for data collection in wireless sensor networks: mobility-aware sink selection," The Open Automation and Control Systems Journal, vol. 8, no. 1, pp. 35-46, 2016. 
[11] C. C. Grant, A. Jones, A. Hamins, and N. Bryner, "Realizing the vision of smart fire fighting," IEEE Potentials, vol. 34, no. 1, pp. 35-40, 2015.

[12] A. Paul, A. Ahmad, M. M. Rathore, and S. Jabbar, "Smartbuddy: defining human behaviors using big data analytics in social internet of things," IEEE Wireless Communications, vol. 23, no. 5, pp. 68-74, 2016.

[13] C. Chatrapathi, M. N. Rajkumar, and V. Venkatesakumar, "VANET based integrated framework for smart accident management system," in Proceedings of the IEEE International Conference on Soft-Computing and Network Security (ICSNS '15), Coimbatore, India, February 2015.

[14] A. H. Levis and L. W. Wagenhals, "C4ISR architectures: I. Developing a process for C4ISR architecture design," Systems Engineering, vol. 3, no. 4, pp. 225-247, 2000.

[15] D. Chen, G. Doumeingts, and F. Vernadat, "Architectures for enterprise integration and interoperability: past, present and future," Computers in Industry, vol. 59, no. 7, pp. 647-659, 2008.

[16] H. Park, H. Kim, H. Joo, and J. Song, "Recent advancements in the Internet-of-Things related standards: a oneM2M perspective," ICT Express, vol. 2, no. 3, pp. 126-129, 2016.

[17] D. Evans, "The Internet of Things: How the Next Evolution of the Internet Is Changing Everything," 2017, http://www.cisco .com/c/dam/en_us/about/ac79/docs/innov/IoT_IBSG_0411FINAL .pdf.

[18] E. Avelar, L. Marques, D. Dos Passos, R. Macedo, K. Dias, and M. Nogueira, "Interoperability issues on heterogeneous wireless communication for smart cities," Computer Communications, vol. 58, pp. 4-15, 2015.

[19] M. Ganzha, M. Paprzycki, W. Pawłowski, P. Szmeja, and K. Wasielewska, "Semantic interoperability in the internet of things: an overview from the INTER-IoT perspective," Journal of Network and Computer Applications, vol. 81, pp. 111-124, 2017.

[20] R. Ambrosio and S. Widergren, "A framework for addressing interoperability issues," in Proceedings of the IEEE Power Engineering Society General Meeting (PES '07), Tampa, Fla, USA, June 2007.

[21] K. R. Malik, T. Ahmad, M. Farhan et al., "Big-data: transformation from heterogeneous data to semantically-enriched simplified data," Multimedia Tools and Applications, vol. 75, no. 20, pp. 12727-12747, 2016.

[22] J. Santos, J. J. P. C. Rodrigues, B. M. C. Silva, J. Casal, K. Saleem, and V. Denisov, "An IoT-based mobile gateway for intelligent personal assistants on mobile health environments," Journal of Network and Computer Applications, vol. 71, pp. 194-204, 2016.

[23] A. Yachir, B. Djamaa, A. Mecheti, Y. Amirat, and M. Aissani, "A comprehensive semantic model for smart object description and request resolution in the internet of things," Procedia Computer Science, vol. 83, pp. 147-154, 2016.

[24] P. P. Jayaraman, D. Palmer, A. Zaslavsky, and D. Georgakopoulos, "Do-it-Yourself Digital Agriculture applications with semantically enhanced IoT platform," in Proceedings of the 10th IEEE International Conference on Intelligent Sensors, Sensor Networks and Information Processing (ISSNIP '15), Singapore, April 2015.

[25] P. Desai, A. Sheth, and P. Anantharam, "Semantic gateway as a service architecture for IoT interoperability," in Proceedings of the 3rd IEEE International Conference on Mobile Services (MS '15), pp. 313-319, New York, NY, USA, July 2015.

[26] A. Gyrard and M. Serrano, "Connected smart cities: interoperability with SEG 3.0 for the internet of things," in Proceedings of the 30th IEEE International Conference on Advanced Information Networking and Applications Workshops (WAINA '16), pp. 796-802, March 2016.

[27] H. Fotouhi, A. Causevic, M. Vahabi, and M. Björkman, "Interoperability in heterogeneous low-power wireless networks for health monitoring systems," in Proceedings of the in IEEE International Conference on Communications Workshops (ICC '06), 2016.

[28] E. Mingozzi, G. Tanganelli, C. Vallati, B. Martinez, I. Mendia, and M. Gonzalez-Rodriguez, "Semantic-based context modeling for quality of service support in IoT platforms," in Proceedings of the 17th International Symposium on a World of Wireless, Mobile and Multimedia Networks (WoWMoM '16), Coimbra, Portugal, June 2016.

[29] C. Pereira, A. Pinto, A. Aguiar, P. Rocha, F. Santiago, and J. Sousa, "IoT interoperability for actuating applications through standardised M2M communications," in Proceedings of the 17th International Symposium on a World of Wireless, Mobile and Multimedia Networks (WoWMoM '16), Coimbra, Portugal, June 2016.

[30] P. P. Jayaraman, C. Perera, D. Georgakopoulos, S. Dustdar, D. Thakker, and R. Ranjan, "Analytics-as-a-service in a multicloud environment through semantically-enabled hierarchical data processing," Software-Practice and Experience, 2016.

[31] N. Shariatzadeh, T. Lundholm, L. Lindberg, and G. Sivard, "Integration of digital factory with smart factory based on internet of things," Procedia CIRP, vol. 50, pp. 512-517, 2016.

[32] P. Buneman and S. Staworko, "RDF graph alignment with bisimulation," Proceedings of the VLDB Endowment, vol. 9, no. 12, pp. 1149-1160, 2016.

[33] W. Zheng, L. Zou, W. Peng, X. Yan, S. Song, and D. Zhao, "Semantic SPARQL similarity search over RDF knowledge graphs," Proceedings of the VLDB Endowment, vol. 9, no. 11, pp. 840-851, 2016. 


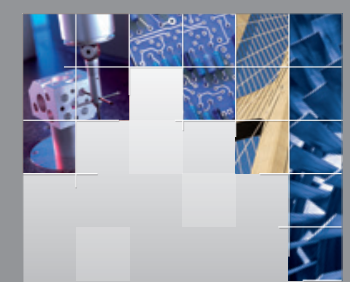

\section{Enfincering}
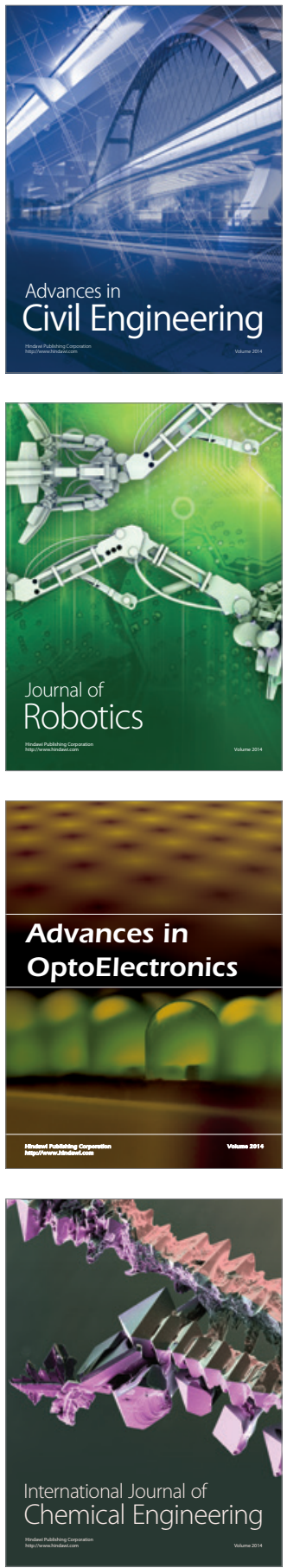

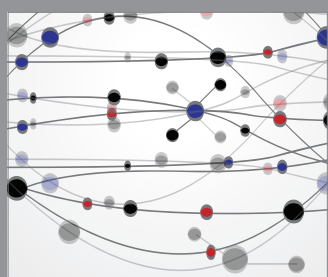

The Scientific World Journal

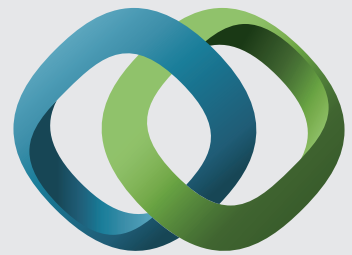

\section{Hindawi}

Submit your manuscripts at

https://www.hindawi.com
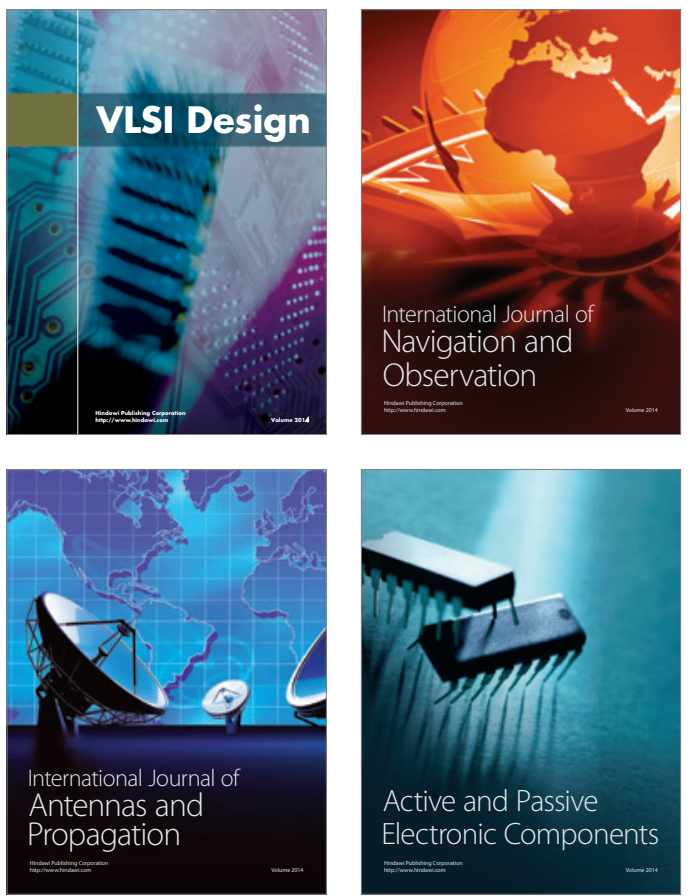
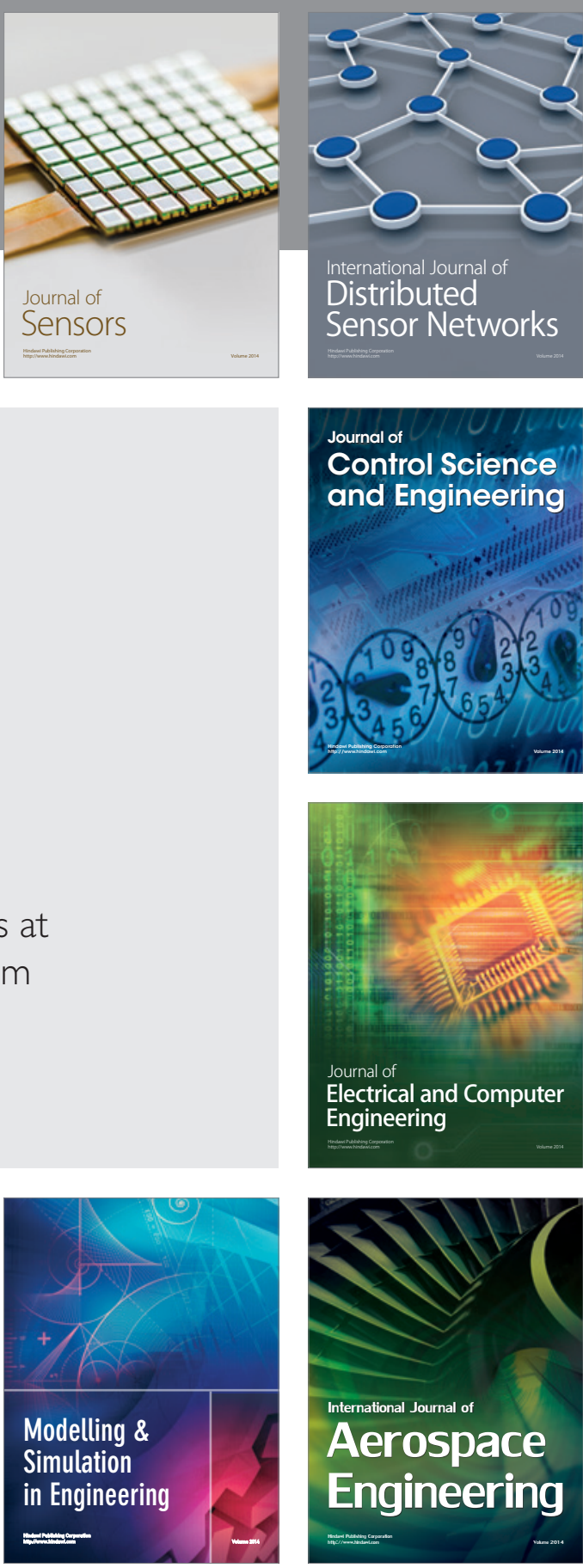

International Journal of

Distributed

Sensor Networks

$-$

Joumal of

Control Science

and Engineering
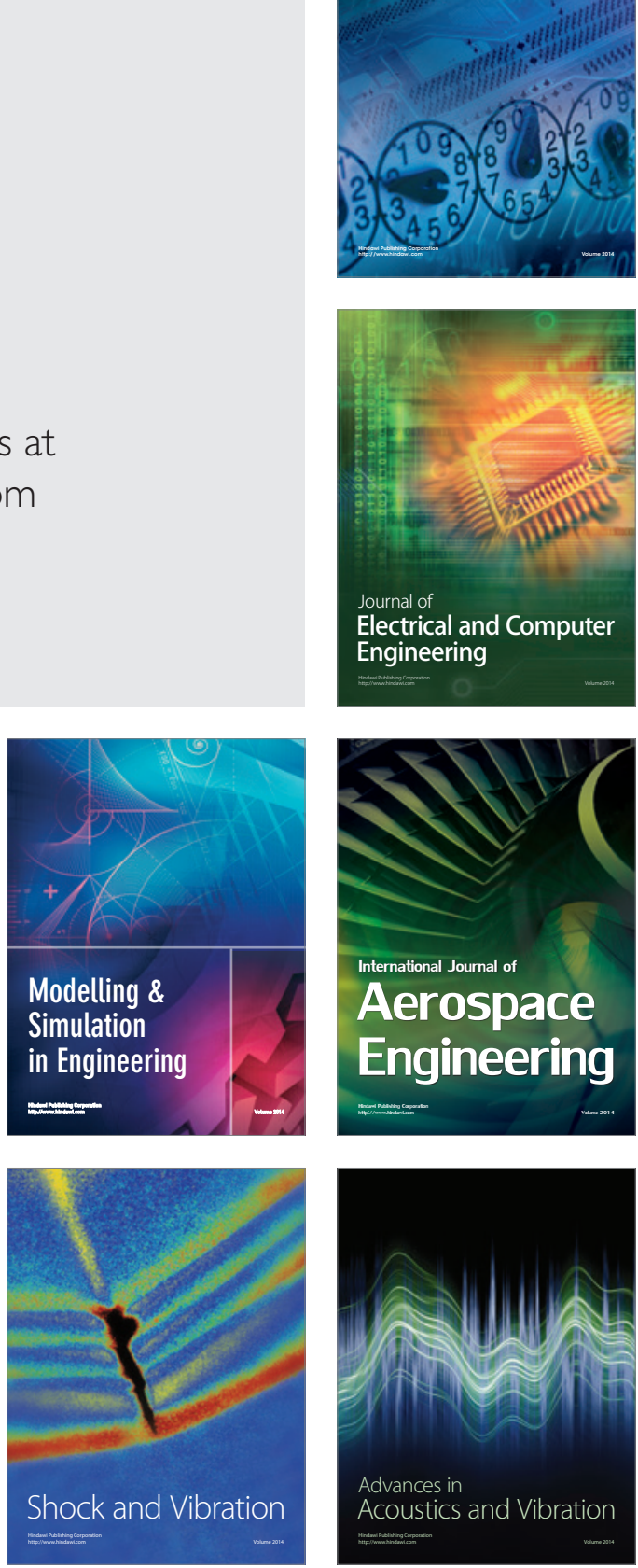